\title{
Oral manifestation of histoplasmosis on the palate*
}

Barbara Capitanio de Souza ${ }^{1}$

\author{
Maria Cristina Munerato ${ }^{2,3}$
}

DOI: http://dx.doi.org/10.1590/abd1806-4841.20175751

\begin{abstract}
This case report describes an uncommon manifestation of histoplasmosis on the soft palate. The importance of appropriate treatment and follow-up in complex cases is emphasized, especially in patients with chronic diseases. Oral lesions may occur as multiple, granular and painful ulcers, as well as verrucous growths. Lesions may also be deep, with infiltrative edges and erythematous or with white areas, accompanied by local lymphadenopathy, resembling a carcinoma on clinical examination. In this sense, a fast and accurate diagnosis is essential to the success of treatment of oral histoplasmosis.
\end{abstract}

Keywords: Histoplasmosis; Oral manifestations; Oral ulcer

\section{INTRODUCTION}

Histoplasmosis is a systemic disease caused by Histoplasma capsulatum, a dimorphic fungus that can cause multiple clinical manifestations. It is more prevalent in some regions in the North, Central, and Latin America, as well as in Africa, and is typically found in tropical and temperate rural areas. The disease is acquired by inhalation of dust particles from the soil, contaminated with bat or bird feces, which contain fungal spores, the infectious form of the microorganism. Lesions on the oral mucosa are rather rare, and can be a presentation of disseminated histoplasmosis or the only site involved. More severe and generalized cases can be observed in patients with immune deficiencies, like the elderly, or HIV positive individuals. ${ }^{1}$ Histoplasmosis may also be associated with chronic obstructive pulmonary disease (COPD) and to the use of corticosteroid or immunosuppressants required for organ transplant patients. ${ }^{2}$ We herein report a case of an oral lesion of histoplasmosis.

\section{CASE REPORT}

An 81-year-old male Caucasian patient was referred to our unit with a lesion on the soft palate that had appeared three months before (Figure 1). Past medical history included smoking, COPD, asthma, arthrosis, and a prostate cancer, already treated. For his pulmonary conditions, he needed to use immunosuppressant medication regularly. Physical examination revealed a $25-\mathrm{mm}$ granular ulceration with red and yellow dots in the hard-soft palate transition zone. With an incisional biopsy, histoplasmosis was diagnosed. The slides with the specimens were stained with hematoxylin-eosin (HE) and Gomori's methenamine-silver (GMS), confirming the presence of the fungus (Figure 2). The patient was referred to assess pulmonary involvement, and there was no evidence of active pleuropulmonary lesions (Figure 3). Itraconazole $200 \mathrm{mg}$ /day was prescribed for the treatment of oral histoplasmosis, with significant improvement after 30 days (Figure 4). However, 60 days after starting the treatment, the patient's respirato-

Work submitted on 03.01.2016

Approved by the Advisory Board and accepted for publication on 20.06.2016

* Work performed at the Oral Medicine Unit - Hospital de Clínicas de Porto Alegre - Universidade Federal do Rio Grande do Sul (HCPA-UFRGS) - Porto Alegre (RS), Brazil.

Financial Support: None.

Conflict of Interests: None.

Department of Oral Medicine - Centro de Especialidades Odontológicas (Ceo I) - Alvorada (RS), Brazil.

Department of Conservative Dentistry - Faculdade de Odontologia - Universidade Federal do Rio Grande do Sul (UFRGS) - Porto Alegre (RS), Brazil.

Oral Medicine Unit - Hospital de Clínicas de Porto Alegre (HCPA) - Porto Alegre (RS), Brazil. 


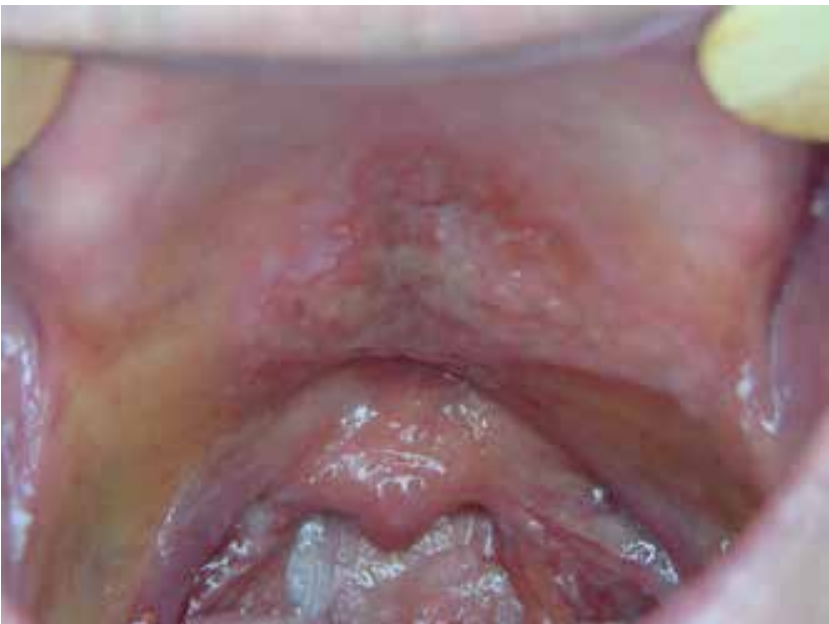

Figure 1: Clinical aspect of the histoplasmosis lesion: granular ulcer with red and yellow dots in the hard-soft palate ry condition worsened, with dyspnea on exertion. He quickly evolved to acute respiratory failure and sepsis of unknown origin. With no response to the treatment, the patient eventually died.

\section{DISCUSSION}

Histoplasmosis is a systemic fungal infection caused by inhalation of H.capsulatum spores, a dimorphic fungus held responsible for a variety of clinical manifestations. It can be found in hot and humid environments, with a predilection for areas with acid soils. After contagion of the airways, the microconidia are phagocytosed by macrophages and start to replicate intracellularly. Hematogenous spread occurs within two weeks after infection. In immunocompetent patients, the macrophages have a fungicidal role, phagocytizing H.capsulatum and controlling the disease. ${ }^{3}$

Histoplasmosis has been reported in all continents, particularly in temperate regions. It is an endemic disease in some areas such as Ohio, Mississippi River Valley, Central and South America
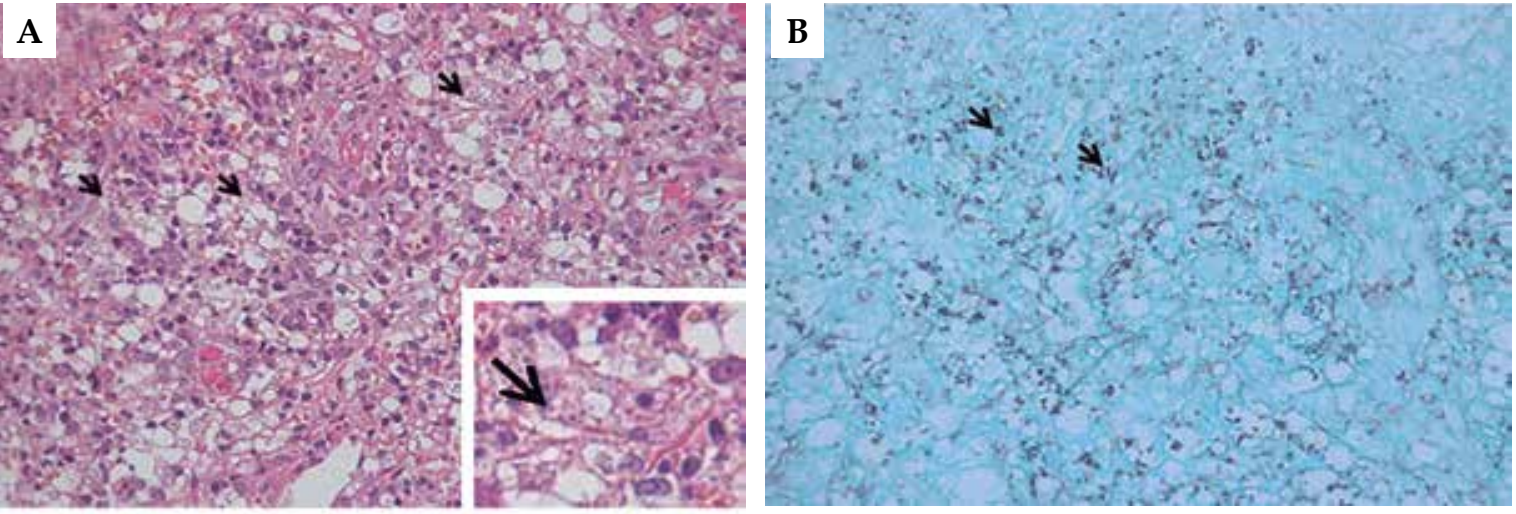

FIGURE 2: Histopathological aspects of histoplasmosis. A: microphotograph showing diffuse infiltrate of epithelioid macrophages containing $H$. capsulum (arrow). Presence of lymphoplasmacytic inflammatory infiltrate with polymorphonuclear neutrophils (Hematoxylin \& eosin, X400). B: microphotograph showing small particles of H. capsulatum measuring between 1 and $2 \mu \mathrm{m}$ (arrow). (GMS, X200)
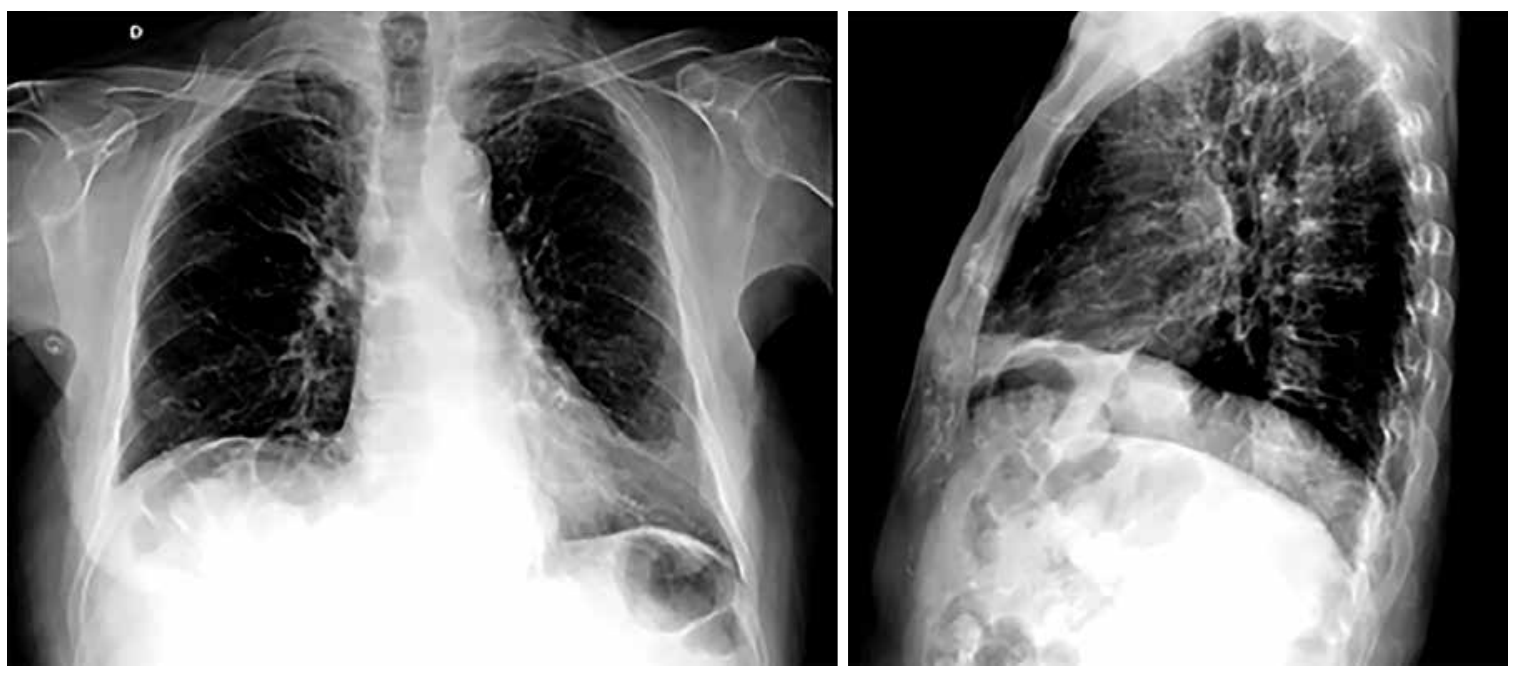

FIGURE 3: Radiograph revealed micronodules and fibroatelectatic changes, which are nonspecific and may be residual, associated with smoking and COPD. There is no evidence of active pleuropulmonary lesions 


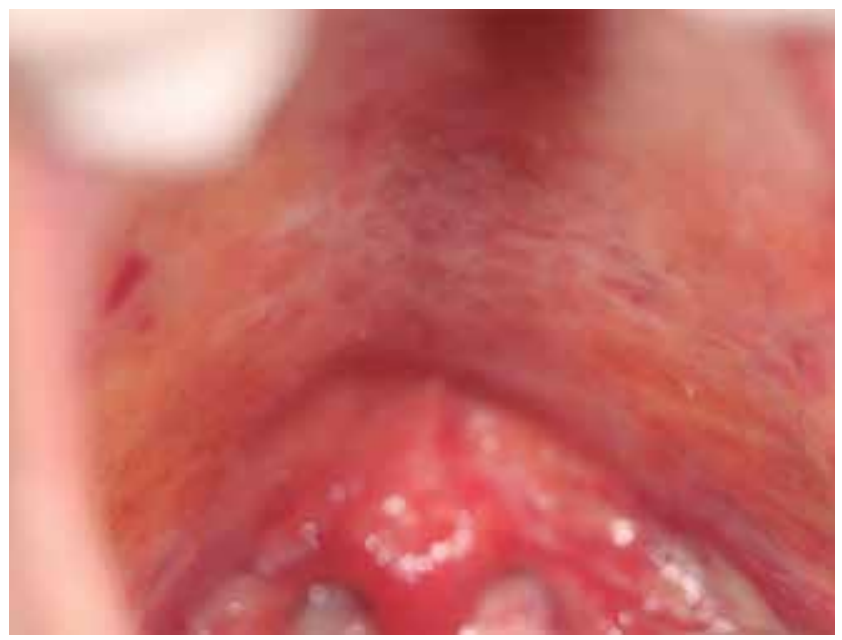

Figure 4: Clinical aspect of the lesion 30 days after starting treatment

and Africa. ${ }^{3}$ In Brazil, endemic regions include the states of Rio de Janeiro, Minas Gerais, Ceará and Rio Grande do Sul, in the Jacuí River Valley in particular. ${ }^{4}$

Most oral lesions occur in the disseminated form of the disease and may affect any area of the oral cavity. ${ }^{5}$ The manifestation will be a function of the number of spores inhaled, of the host's immune system, and of the fungal strains involved. Nevertheless, the condition can also affect immunocompetent individuals, manifesting as an asymptomatic self-limited flu-like process. Several case reports describe the occurrence of severe extra-pulmonary lesions in children and in patients with underlying conditions. ${ }^{6}$ It can be latent for years. ${ }^{7}$ It is possible that the genetic differences between the strains can alter the pathogenesis and the clinical manifestations of histoplasmosis. This disease is rarely fatal, and is usually manifested as a self-limited lung infection. Children and elderly patients that have chronic diseases may develop the more severe form, leading to death in some cases, mainly due to the dissemination of the fungus. ${ }^{3}$
Oral histoplasmosis lesions normally occur as granular ulcerations, multiple painful ulcers and verrucous growths, deep ulcers surrounded by infiltrative edges with erythematous or white areas with irregular surfaces, as hardened and irregular nodular lesions accompanied by local lymphadenopathy, mimicking other infectious diseases or malignant tumors. ${ }^{4}$ The sites commonly involved in the oral cavity are the tongue, the palate, the oral mucosa, the gingivae, and the pharynx. Differential diagnosis should include squamous cell carcinoma, hematologic malignancies, tuberculosis, other deep fungal infections, oral lesions observed with Crohn's disease, necrotizing sialometaplasia of the palate, and chronic traumatic ulcers. ${ }^{5}$

Biopsy can be the most efficient method to obtain the diagnosis of histoplasmosis, allowing prompt treatment. Special stains, such as GMS, are useful for this end. ${ }^{8}$ Currently, treatment of histoplasmosis with amphotericin B is only recommended as the initial therapy in more severe cases. With improvement of the clinical condition, replacement with an azole agent is indicated. Itraconazole is the drug of choice to treat mild and moderate histoplasmosis. ${ }^{9}$ Antifungals are used in severe acute histoplasmosis, and all chronic and disseminated cases of the disease. For those who do not tolerate amphotericin B, itraconazole is an effective treatment and can be prescribed as prophylaxis in patients with advanced HIV infection ${ }^{5}$. New drugs such as voriconazole and posaconazole are also effective against the disease. Patients that were initially treated with fluconazole can present cross-resistance with voriconazole. ${ }^{9}$ However, even with adequate treatment, there is risk of failure and relapse, which will lead to prolonged treatment.

In summary, histoplasmosis is an opportunistic fungal disease that often presents in the oral cavity in immunodeficient individuals; in addition, other conditions of the patients may contribute to the infection. Histoplasmosis should be included in the differential diagnosis of oral ulcers, regardless of pulmonary symptoms or the fact that the patient lives in a non-endemic area. The early and accurate diagnosis of histoplasmosis is of the utmost importance for the treatment and cure of the disease.]

\section{REFERENCES}

1. Matulionyte R, Garbino J, Uçkay I, Lambert JF, Hirschel B.. Disseminated histoplasmosis in an HIV-infected patient discovered by routine blood smear staining. Eur J Clin Microbiol Infect Dis. 2005;24:361-3.

2. Ryu JH, Swensen SJ. Cystic and cavitary lung diseases: focal and diffuse. Mayo Clin Proc. 2003;78:744-52.

3. Mignogna MD, Fedele S, Lo Russo L, Ruoppo E, Lo Muzio L. A case of oral localized histoplasmosis in an immunocompetent patient. Eur J Clin Microbiol Infect Dis. 2001;20:753-5.

4. Kauffman CA. Histoplasmosis: a clinical and laboratory update. Clin Microbiol Rev. 2007;20:115-32.

5. Vidyanath S, Shameena P, Sudha S, Nair RG. Disseminated Histoplasmosis with oral and cutaneous manifestations. J Oral Maxillofac Pathol. 2013;17:139-42.

6. Siraj F, Manucha V. Pharyngeal histoplasmosis presenting as a tumor mass in an immunocompetent host. J Glob Infect Dis. 2010;2:70-1.

7. Torres-Rodríguez JM, Segura-Roca G, Coll J. Histoplasmosis 45 years after infection in an immunocompetent man. Rev Iberoam Micol. 2009;26:244-6.
8. Yang B, Lu L, Li D, Liu L, Huang L, Chen L, et al. Colonic involvement in disseminated histoplasmosis of an immunocompetent adult: case report and literature review. BMC Infect Dis. 2013;13:143.

9. Wheat LJ, Connolly P, Smedema M, Durkin M, Brizendine E, Mann P, Patel R McNicholas PM, Goldman M. Activity of newer triazoles against Histoplasma capsulatum from patients with AIDS who failed fluconazole. J Antimicrob Chemother. 2006;57:1235-9.

MAILING ADDRESS:

Maria Cristina Munerato

Rua Ramiro Barcelos, 2350

90035-007 Porto Alegre, RS.

Brazil

E-mail:momunerato@gmail.com

How to cite this article: Souza BC, Munerato MC. Oral manifestation of histoplasmosis on the palate. An Bras Dermatol. 2017;92(5 Suppl 1): 107-9. 